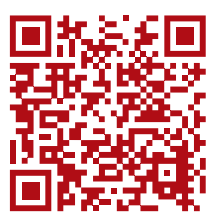

\title{
Manejo de fracturas faciales en el paciente adulto mayor: experiencia de la clínica de cirugía ortognática y trauma facial del Hospital «Dr. Manuel Gea González»
}

\author{
Handling of facial fractures in the elderly patient: \\ experience of the orthognathic surgery and facial trauma \\ clinic of the «Dr. Manuel Gea González» Hospital
Dr. Federico Iñigo-Arroyo,* Dr. Jacobo Felemovicius-Hermangus, ${ }^{\ddagger}$ Dr. Enrique Chávez-Serna,* Dra. Andrea Carrillo-Romero*

Palabras clave: Trauma facial, fracturas faciales, adulto mayor, caídas.

Keywords: Facial trauma, facial fractures, elderly, falls.

\footnotetext{
* Servicio de Cirugía Plástica y Reconstructiva. División de Estudios de Posgrado, Universidad Nacional Autónoma de México. * Jefe de la Clínica de Cirugía Ortognática y Trauma Facial.
}

División de Cirugía Plástica y Reconstructiva, Hospital General «Dr. Manuel Gea González». Cuidad de México. México.

Recibido: 23 diciembre 2020 Aceptado: 19 abril 2021

\section{RESUMEN}

Debido al aumento de la expectativa de vida, se observan con mayor frecuencia fracturas faciales en el adulto mayor. El trauma facial disminuye significativamente la calidad de vida del paciente geriátrico. El objetivo de este trabajo es describir la experiencia en el manejo de las fracturas faciales en el adulto mayor de la clínica de cirugía ortognática y trauma facial del Hospital General «Dr. Manuel Gea González». Se realizó un estudio retrospectivo, de 2013 a 2019, de los expedientes de pacientes mayores de 65 años con fracturas faciales. Las variables consideradas fueron edad, sexo, etiología, tercio craneofacial afectado, hueso afectado, tratamiento y complicaciones asociadas. Se incluyeron 74 pacientes en total: 45 hombres (61\%) y 29 mujeres (39\%). La edad promedio fue de 76.2 años (DE $=8.4)$. La causa más frecuente fueron las caídas en 52 casos $(70 \%)$. Las fracturas de piso de órbita fueron las más frecuentes, con 26 casos (35\%). El tratamiento fue conservador en 51 casos (69\%) y quirúrgico en 13 casos (31\%). En 12 casos (16\%) se presentaron complicaciones, las más frecuentes fueron las parestesias en cinco casos (42\%). Concluimos que las caídas representan la principal causa de fracturas faciales en el adulto mayor y generalmente, por la baja energía asociada, no ocasionan lesiones severas, por lo cual, en la mayoría de los casos se puede ofrecer tratamiento conservador con excelentes resultados.

\section{ABSTRACT}

Due to the increase in life expectancy, facial fractures in the elderly occur more frequently. Facial trauma gives rise to a significant decrease in the quality of life of the geriatric patient. The objective of this work is to describe the experience in handling facial fractures in the elderly at the orthognathic surgery and facial trauma clinic of the General Hospital «Dr. Manuel Gea González». A retrospective study was carried out, from 2013 to 2019, of the records of patients with facial fractures older than 65 years. The variables considered were: age, sex, etiology, affected craniofacial third, damaged bone, treatment and associated complications. A total of 74 patients were included: 45 men (61\%) and 29 women (39\%). The mean age was 76.2 years $(S D=8.4)$. Falls were most recurrent cause in 52 cases $(70 \%)$. Orbit floor fractures were the most frequent, with 26 cases (35\%). Treatment was conservative in 51 cases (69\%) and surgical in 13 cases (31\%). In 12 cases (16\%) there were complications, the most persistent were paresthesia in five cases (42\%). We conclude that falls represent the main cause of facial fractures in the elderly and are generally, related to reduced low energy. They do not cause severe injuries; therefore, in most cases, conservative treatment can be given with excellent results.

Citar como: Iñigo-Arroyo F, Felemovicius-Hermangus J, Chávez-Serna E, Carrillo-Romero A. Manejo de fracturas faciales en el paciente adulto mayor: experiencia de la clínica de cirugía ortognática y trauma facial del Hospital «Dr. Manuel Gea González». Cir Plast. 2021; 31 (3): 97-101. https://dx.doi.org/10.35366/103710 


\section{INTRODUCCIÓN}

$\mathrm{D}_{\mathrm{t}}^{\mathrm{s}}$ ebido al aumento significativo en la expectativa de vida en las últimas décadas, las fracturas faciales en la población mayor de 65 años se presentan en la actualidad con mayor frecuencia, por lo tanto, es importante conocer el acceso y tratamiento de esta patología, con el objetivo de mejorar los resultados funcionales en este grupo de edad. ${ }^{1}$ Las fracturas faciales se pueden presentar de diferentes formas, las cuales, dependiendo de su severidad, pueden ocasionar un deterioro importante en la calidad de vida de los individuos y tienen un mayor impacto en el adulto mayor. Las caídas son consideradas en la actualidad como un problema de salud pública y representan el mecanismo más frecuente de lesión en este grupo de edad, además, son una de las principales causas de visita en los servicios de urgencia, lo cual genera un impacto importante en los costos de los servicios de salud. En una revisión sistemática realizada en Estados Unidos de Norteamérica, se encontró que los costos en salud asociados a las caídas representan de 0.85 a 1.5\% del total de los costos en los servicios en salud. ${ }^{2}$ De acuerdo con la Encuesta Nacional de la Dinámica Demográfica (ENADID), realizada por el INEGI (Instituto Nacional de Estadística y Geografía) en 2018, el número de personas mayores de 60 años incluye a 15.4 millones, que corresponde a $12.4 \%$ de la población. ${ }^{3}$ Desafortunadamente, en México se desconoce la cifra exacta de pacientes que presentan fracturas faciales, los tipos de fractura más comunes, su tratamiento y las secuelas que éstas generan en el paciente mayor de 65 años. En Estados Unidos de Norteamérica, la prevalencia de las fracturas faciales es de $4.9 \%$ y son las fracturas menos frecuentes, en contraste con las fracturas de cadera (34.1\%); sin embargo, se ha visto un aumento importante en los últimos años, probablemente debido a una mayor participación del adulto mayor en actividades recreacionales y laborales. ${ }^{4}$ El trauma facial genera una mayor morbimortalidad al generar efectos negativos en la respiración, deglución, habla, masticación y visión, lo cual se relaciona con disminución en la función e independencia del paciente geriátrico. El objetivo de este artículo es proporcionar un panorama general de la etiología, patrón, tratamiento y complicaciones de las fracturas faciales en el paciente geriátrico en un centro de referencia en trauma facial y cirugía ortognática.

\section{MATERIAL Y MÉTODOS}

Diseñamos un estudio observacional, retrospectivo, transversal y descriptivo, en el cual se incluyeron todos los expedientes de pacientes mayores de 65 años con presencia de fracturas faciales, tratados en el Hospital General «Dr. Manuel Gea González», en el periodo de enero de 2013 a julio de 2019. La información se extrajo de los expedientes clínicos y fotográficos de los pacientes y fue ingresada en una hoja de captura de datos en la que se incluyeron variables demográficas de los pacientes, etiología de la fractura, hueso afectado, tratamiento establecido y complicaciones asociadas al tipo de tratamiento. Se realizó un análisis descriptivo de las características de la población con el paquete estadístico SPSS versión 25, en el cual las variables categóricas se reportan en porcentajes y las continuas como promedio ( \pm desviación estándar) o mediana (rango intercuartílico [IQR]) de acuerdo con su distribución.

\section{RESULTADOS}

Se incluyeron 74 pacientes en total, 45 hombres (61\%) y 29 mujeres (39\%). La edad promedio fue de $76.2 \pm 8.4$ años, con un rango de 65 a 100 años.

En cuanto a la etiología, la causa más frecuente observada en este estudio fueron las caídas en 52 casos (70\%), seguido de la agresión por terceras personas en 18 (24\%) y en último lugar los accidentes automovilísticos en cuatro casos (5\%).

Las fracturas fueron clasificadas de acuerdo con el tercio del macizo facial y el hueso afectado. El tercio medio representó el más frecuente con 61 casos (82\%), seguido del tercio inferior con siete $(10 \%)$ y en último lugar el tercio superior con tres (4\%). Las fracturas de piso de órbita fueron las más frecuentes, con 26 casos (35\%), seguido de las fracturas de pared de órbita, con 17 (23\%), fracturas del cóndilo mandibular siete (10\%) y de los huesos propios 
Tabla I: Presentación de las fracturas faciales.

\begin{tabular}{llc} 
Tercio & Hueso afectado & $\mathrm{n}(\%)$ \\
\hline Superior & Frontal & $3(4)$ \\
Medio & Piso de órbita & $26(35)$ \\
& Pared de órbita & $17(23)$ \\
& Nasal & $7(10)$ \\
& Maxilar & $5(7)$ \\
& Arco cigomático & $4(5)$ \\
& Etmoidal & $1(1)$ \\
\multirow{2}{*}{ Inferior } & Órbito-cigomático & $1(1)$ \\
Panfacial & Mandibular & $7(10)$ \\
& Nasal-frontal-mandibular & $3(4)$ \\
\hline
\end{tabular}

de la nariz siete $(10 \%)$, representaron las terceras más frecuentes. En la Tabla 1 se presentan todas las fracturas reportadas en este estudio.

El tratamiento fue conservador en 51 pacientes (69\%) y quirúrgico en 13 (31\%). El tratamiento conservador se realizó con analgésico, antibiótico y seguimiento estricto del paciente en consultas subsecuentes. El tratamiento quirúrgico se individualizó dependiendo del tipo de fractura. En la mayoría de los casos se realizó reducción abierta más fijación interna (RAFI), más aseo, desbridamiento y cierre primario de la herida.

En 12 (16\%) se presentaron complicaciones asociadas al tratamiento, de las cuales, las más frecuentes fueron las parestesias en cinco casos $(42 \%)$, seguido de la diplopía en cuatro (33\%); en tercer lugar, la exposición de material de osteosíntesis en dos (17\%) y, en último lugar, la infección del sitio quirúrgico en un caso $(8 \%)$.

\section{DISCUSIÓN}

Se espera en forma global un aumento constante de la población mayor de 65 años en los próximos años, esto debido al aumento significativo en la expectativa de vida y uno de los principales factores es la disminución en la mortalidad de las enfermedades crónico-degenerativas e infectocontagiosas. En México, en el año 1990, la esperanza de vida en promedio era de 70 años para ambos sexos; para 2030 se espera que llegue a 77 años. ${ }^{5} \mathrm{El}$ trauma en general en este grupo de edad es secundario a las caídas, contrario a lo que sucede en población más joven, donde los accidentes automovilísticos representan la causa más frecuente. Por otro lado, la recuperación del trauma en estos pacientes es más lenta, generando más secuelas, lo cual puede condicionar un deterioro súbito en el estado de salud y la calidad de vida en estos pacientes.

En este estudio encontramos al sexo masculino con mayor predisposición (61\%) de presentar fracturas faciales, a diferencia de lo reportado en estudios similares, donde el sexo femenino tiene más riesgo de presentar caídas $y$, por ende, fracturas faciales, ${ }^{6,7}$ probablemente debido a la mayor participación en el ámbito laboral del sexo masculino en el país. De acuerdo con la encuesta intercensal realizada en 2015 por el Instituto Nacional de Estadística y Geografía (INEGI), del total de la población de 60 años y más, $27.2 \%$ corresponde a personas económicamente activas y por sexo resalta que los hombres representan $43.5 \%$, en comparación con las mujeres $(13.1 \%){ }^{8}$

Las caídas en nuestro estudio fueron la causa más frecuente de fracturas faciales $(70 \%)$, similar a lo reportado en la literatura internacional. ${ }^{6}$ Causas menos frecuentes representaron la agresión por terceras personas y los accidentes automovilísticos, contrario a lo que sucede en población más joven. Algunos factores de riesgo para presentar caídas son: cambios asociados a la edad como pérdida de fuerza, balance y comorbilidades como pérdida mineral ósea, disminución de la agudeza visual, demencia y polifarmacia. ${ }^{9,10}$ En México, de acuerdo con la Guía de Práctica Clínica, la prevalencia de caídas en el adulto mayor oscila de 30 a $50 \%$, con una incidencia anual de 25 a $50 \%$, de las cuales, de 10 a $25 \%$ provocan fracturas, requiriendo hospitalización en 5\%, con una mortalidad de $30 \%$ en personas mayores de 65 años. ${ }^{11}$ Las estructuras que se lesionan con mayor frecuencia debido a las caídas son: cadera 50\%, cabeza y cara $24 \%$, mano $10 \%$, hombro $9 \%$ y tobillo $9 \% .{ }^{9}$ Pese a estos reportes, no se conoce la incidencia exacta de las fracturas faciales, cuáles requieren tratamiento quirúrgico, las complicaciones asociadas y el seguimiento funcional a largo plazo, incluyendo las secuelas que éstas generan. 
En este estudio y de acuerdo con otros similares, el tercio medio de la cara representa el sitio que con más frecuencia se ve afectado, $6,12,13$ siendo $85 \%$ del total de fracturas presentadas, en comparación con el tercio superior e inferior, explicado probablemente por la cinemática del trauma y la energía de éste en las caídas (Tabla 1). A diferencia de lo que sucede en otros grupos poblaciones, las fracturas de tercio superior e inferior en el paciente geriátrico son menos comunes, ya que éstas requieren de mayor energía y en su mayoría son generadas por accidentes automovilísticos o traumas de alto impacto. Dentro de las fracturas de tercio medio encontramos que las más comunes fueron las fracturas del piso de la órbita, que representaron 35\%, seguido de las fracturas de la pared de la órbita en 23\% $y$, en tercer lugar, las fracturas nasales en $10 \%$. En diferentes estudios se reporta a las fracturas nasales como las fracturas faciales más frecuentes en población adulta y joven. ${ }^{14}$ En contraste con nuestros hallazgos, las fracturas nasales representaron el tercer lugar en frecuencia; sin embargo, se asocian de manera más común con fracturas de órbita y maxilares, a diferencia de la población joven, donde se asocian con fracturas mandibulares, las cuales son poco frecuentes en la población geriátrica (Tabla 1). ${ }^{6}$

El tratamiento proporcionado en este estudio en su mayoría fue conservador (69\%), con antibioticoterapia profiláctica y seguimiento estrecho en consultas subsecuentes, similar a lo reportado por diferentes autores. ${ }^{15-17}$ Por otro lado, el tratamiento quirúrgico (31\%), se limitó a fracturas que generaban deficiencia funcional, dislocación significativa de la fractura, riesgo de sepsis o falta de consolidación. Para este tipo de fracturas realizamos la reducción abierta y fijación interna en el quirófano con placas y tornillos. La decisión de llevar a cabo el tratamiento quirúrgico o conservador en la población geriátrica es difícil y hoy en día no existe un consenso que defina concretamente cuál tiene mejores resultados a largo plazo. Por otro lado y de acuerdo con estudios similares, la mayoría de los expertos opta por el tratamiento conservador por diferentes razones, como comorbilidades en el adulto mayor que confieren mayor riesgo al acto quirúrgico, menor importancia del resultado estético por parte del paciente, la cinemática del trauma que generalmente es de bajo impacto relacionado con las caídas y, por último, que la zona más frecuentemente afectada es el tercio medio que genera menor afectación en la función. ${ }^{6}$ Por lo anterior, se ha observado que existe un menor número de complicaciones cuando se compara el tratamiento quirúrgico con el conservador, teniendo menor costo y riesgo este último, con resultados más favorables. ${ }^{15,17}$ En este estudio, las complicaciones asociadas al tratamiento conservador representaron sólo 16\%, en comparación con $84 \%$ que no presentó. Dentro de éstas, las parestesias y la diplopía representaron las más comunes y conviene resaltar que la mayoría de estas remitió en los primeros seis meses sin tratamiento. La complicación más frecuente asociada al tratamiento quirúrgico fue la exposición del material de osteosíntesis y en un caso la infección del sitio quirúrgico, las cuales fueron manejadas con antibióticos, retiro del material y aseo más desbridamiento quirúrgico.

\section{Limitaciones}

Este estudio cuenta con las limitaciones de ser retrospectivo. No se reporta el resultado funcional a largo plazo, comparando el tratamiento quirúrgico con el conservador, pero es el primer trabajo relacionado con fracturas faciales en el adulto mayor en el país, lo cual nos permitirá realizar ensayos prospectivos con la finalidad de contar con algoritmos de tratamiento que mejoren el pronóstico de los pacientes.

\section{CONCLUSIÓN}

De acuerdo con los hallazgos reportados y con lo publicado en la literatura internacional, concluimos que las fracturas faciales en la tercera edad son un problema de salud pública que se presenta con mayor frecuencia debido al aumento en la expectativa de vida en el país y el mundo. Las caídas representan la principal causa, por lo cual la prevención de éstas continúa siendo el pilar del tratamiento primario. Las fracturas faciales en el adulto mayor acontecen en mayor frecuencia en el tercio medio craneofacial y, por su bajo impacto de fuerza, generalmente no ocasionan lesiones severas, 
por lo cual en su mayoría pueden ser manejadas de manera conservadora, con una baja tasa de complicaciones y resultados favorables.

\section{REFERENCIAS}

1. World Health Organization. World report on ageing and health. WHO 2015: 260.

2. Heinrich S, Rapp K, Rissmann $U$ et al. Cost of falls in old age: a systematic review. Osteoporos Int 2010; 21: 891-902.

3. INEGI [Instituto Nacional de Estadística y Geografía]. Encuesta Nacional de la Dinámica Demográfica (ENADID). 2018. Disponible en: https://www.inegi. org.mx/programas/enadid/2018/

4. Baidwan NK, Naranje SM. Epidemiology and recent trends of geriatric fractures presenting to the emergency department for United States population from year 2004-2014. Public Health 2017; 142:64-69.

5. CONAPO [Consejo Nacional de Población]. Delimitación de las zonas metropolinas de México. 2010. Disponible en: http://www.conapo.gob.mx/es/ CONAPO/Zonas metropolitanas 2010

6. Atisha DM, Burr TVR, Allori AC et al. Facial Fractures in the Aging Population. Plast Reconstr Surg 2016; 137(2): 587-593.

7. Marchini L, Allareddy V. Epidemiology of facial fractures among older adults: A retrospective analysis of a nationwide emergency department database. Dent Traumatol 2019; 35(2): 109-114.

8. INEGI [Instituto Nacional de Estadística y Geografía]. Encuesta Intercensal 2015, base de datos, y Síntesis metodológica y conceptual. 2015. Disponible en: http://www.inegi.org.mx/sistemas/biblioteca/ficha. aspx?upc $=702825078836$

9. Lai SW, Laio KF, Laio CC et al. Polypharmacy correlates with Increased risk for hip fracturesin the elderly: A population-based study. Medicine (Baltimore) 2010; 89: 295-299.

10. Tinetti ME, Doucette J, Claus E et al. Risk factors for serious injury during falls by older persons in the community. J Am Geriatr Soc 1995; 43: 1214-1221.

11. Prevención de Caídas en el Adulto Mayor en el Primer Nivel de Atención México: Secretaria de Salud 2008.

12. Gerbino G, Roccia F, De Gioanni PP et al. Maxillofacial trauma in the elderly. J Oral Maxillofac Surg 1999; 57: 777-782.

13. Goldschmidt MJ, Castiglione CL, Assael LA et al. Craniomaxillofacial trauma in the elderly. J Oral Maxillofac Surg 1995; 53: 1145-1149.

14. Zelken JA, Khalifian S, Mundinger GS, et al. Defining predictable patterns of craniomaxillofacial injury in the elderly: Analysis of 1,047 patients. J Oral Maxillofac Surg 2014; 72: 352-361.

15. Chrcanovic BR, Souza LN, Freire-Maia B et al. Facial fractures in the elderly: a retrospective study in a hospital in Belo Horizonte, Brazil. J Trauma 2010; 69(6): E73-78.

16. Falcone PA, Haedicke GJ, Brooks G et al. Maxillofacial fractures in the elderly. A comparative study. Plast Reconstr Surg 1990; 86: 443-448.

17. Liu FC, Halsey JN, Oleck NC et al. Facial Fractures as a Result of Falls in the Elderly: Concomitant Injuries and Management Strategies. Craniomaxillofac Trauma Reconstr 2019;12(1): 45-53.

Conflicto de intereses: Los autores declaran no tener conflicto de intereses.

Correspondencia:

Dr. Jacobo Felemovicius-Hermangus

Calzada de Tlalpan 4800, Belisario Domínguez Sección 16, 14080, Ciudad de México, México. E-mail: jacofele@gmail.com 